\title{
CHARACTERIZING TOPOLOGIES BY FUNCTIONS
}

\author{
MELVIN C. THORNTON
}

ABSTRACT. The multiplicative structure of the idempotents in the semiring of nonnegative lower semicontinuous functions on a large class of spaces determines the topology of the space.

Nielsen and Sloyer [2] proved that compact $T_{1}$ spaces are homeomorphic iff their semirings of nonnegative lower semicontinuous functions are isomorphic. This note shows that the compact $T_{1}$ assumption is much too strong and that only an isomorphism between the idempotent elements is necessary. Let $L^{+}(X)$ denote the semiring of all nonnegative real-valued lower semicontinuous functions on $X$ with multiplication and addition defined pointwise. A space is a $T_{D}$ space iff the derived set of every point is closed [3].

TheOREM. Let $X$ and $Y$ be $T_{D}$ spaces. Then $X$ is homeomorphic to $Y$ iff the idempotents in $L^{+}(X)$ are isomorphic to the idempotents in $L^{+}(Y)$.

Proof. Let $U(X) \subset L^{+}(X)$ denote the set of idempotents. The elements of $U(X)$ are precisely the characteristic functions of the open sets in $X . U(X)$ is a Brouwerian lattice where $f \leqq g$ iff $f(x) \leqq g(x)$ for all $x$, $f \vee g(x)=\max \{f(x), g(x)\}$, and $f \wedge g(x)=\min \{f(x), g(x)\}$. These lattice operations are determined by the algebra of the semiring as follows. The ordering $f \leqq g$ holds iff $f \cdot g=f$. The meet is $f \wedge g=f \cdot g$. The join of $f$ and $g$ is that unique element $(f \vee g) \in U(X)$ such that $(f \vee g) \cdot f=f$, $(f \vee g) \cdot g=g$, and with the property that if $h \cdot f=f, h \cdot g=g$, then, $(f \vee g) \cdot h=(f \vee g)$. The dual of $U(X), C(X)$, is the lattice of closed sets of $X$ under inclusion and is thus determined by the algebraic structure of the idempotents of $L^{+}(X)$. If $L^{+}(X)$ has its idempotents isomorphic to those of $L^{+}(Y)$ then $C(X)$ is lattice isomorphic to $C(Y)$. Since both spaces are $T_{D}$ spaces, by [3, Theorem 2.1], $X$ is homeomorphic to $Y$. The topology of $X$ can be explicitly recovered from $C(X)$ as in [1].

Received by the editors March 17, 1972.

AMS 1969 subject classifications. Primary 5420; Secondary 5460.

Key words and phrases. Lattice of closed sets, semicontinuous functions.

c) American Mathematical Society 1973 


\section{REFERENCES}

1. D. Drake and W. J. Thron, On the representations of an abstract lattice as the family of closed sets of a topological space, Trans. Amer. Math. Soc. 120 (1965), 57-71. MR 32 \#6390.

2. R. Neilsen and C. Sloyer, Ideals of semi-continuous functions and compactifications of $T_{1}$ spaces, Math. Ann. 187 (1970), 329-331. MR 42 \#8450.

3. W. J. Thron, Lattice-equivalence of topological spaces, Duke Math. J. 29 (1962), 671-679. MR 26 \#4307.

Department of Mathematics, University of Nebraska, Lincoln, Nebraska 68508 